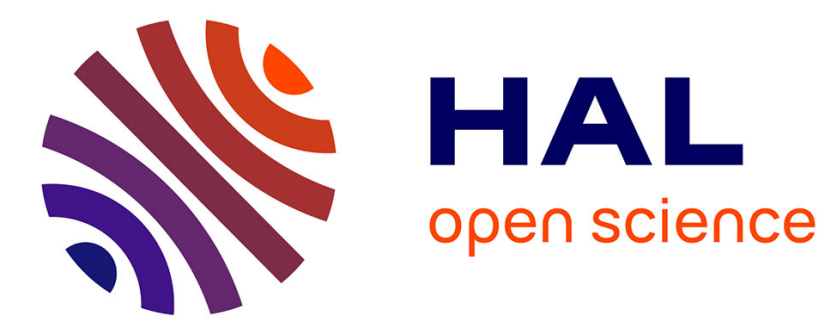

\title{
Une phiale étrusque du Musée de la civilisation gallo-romaine, à Lyon
}

Michel Feugère

\section{To cite this version:}

Michel Feugère. Une phiale étrusque du Musée de la civilisation gallo-romaine, à Lyon. Gallia - Fouilles et monuments archéologiques en France métropolitaine, 1992, 49, pp.1-7. 10.3406/galia.1992.2925 . hal-01914833

\section{HAL Id: hal-01914833 \\ https://hal.science/hal-01914833}

Submitted on 19 Jan 2020

HAL is a multi-disciplinary open access archive for the deposit and dissemination of scientific research documents, whether they are published or not. The documents may come from teaching and research institutions in France or abroad, or from public or private research centers.
L'archive ouverte pluridisciplinaire HAL, est destinée au dépôt et à la diffusion de documents scientifiques de niveau recherche, publiés ou non, émanant des établissements d'enseignement et de recherche français ou étrangers, des laboratoires publics ou privés.

\section{(이) $\$$}

Distributed under a Creative Commons Attribution - NonCommercial - NoDerivatives| 4.0 


\title{
Une phiale étrusque du Musée de la civilisation gallo-romaine, à Lyon
}

\author{
par Michel FEUGÈRE
}

(5) Le début du Premier Age du Fer est marqué à l'ouest et au nord des Alpes par l'apparition de contacts ponctuels entre les mondes étrusque et indigène. Les rapports et les échanges, probablement accaparés par les clệsses privilégiées des sociétés protohistoriques, se traduisent par la présence d'importations luxueuses, généralement des vases en bronze déposés dans les tombes les plus riches du Hallstatt ancien. L'un des marqueurs les plus caractéristiques de ce trafic est une phiale côtelée en tôle de bronze martelée, fabriquée en Étrurie, dès la fin du viI ${ }^{e}$ s., sur des modèles orientaux. Un exemplaire très dégradé, conservé au Musée de Lyon et jusqu'alors interprété comme une trouvaille gallo-romaine, pourrait provenir d'une riche sépulture du début du Premier Age du Fer, découverte anciennement à Lyon ou dans les environs, et non reconnue à l'époque.

To the West and North of the Alps, the beginning of the Earliest Iron Age is characterized by the emergence of intermittent contacts between the Etruscan and indigenous worlds. These relations and exchanges, which were no doubt monopolized by the upper class of the protohistorical societies, are illustrated by the exislence of luxurious imports, generally bronze vessels, placed in the richest graves of the earliest Hallstatt period. One of the most illustrative pieces of these practices is a hammered bronze ribbed phiale, based on an oriental model and produced in Etruria from the late VIIIth C. BC onwards. A much damaged example, preserved in the Museum at Lyons and hitherto considered to be a gallo-roman find, could come from a wealthy tomb of the Early Iron Age, formerly discovered in Lyons or in the neighbouring region, but not identified as such in the time.

Mots clés : phiale, vaisselle de bronze, Étrusque, Hallstatt, importation, Lyon. 
Dans la seconde moitié $d u v^{e} \mathbf{s}$. et au début du $v^{e}$ s. avant notre ère, l'intense activité commerciale déployée par les Grecs de Marseille dans les vallées du Rhône et de la Saône leur assure le monopole d'un marché qu'ils n'entendent pas partager. Sauf exception ${ }^{1}$, tous les produits qui empruntent la voie rhodanienne et atteignent, par les vallées de la Saône et du Doubs, la Bourgogne et la Franche-Comté, sont grecs (ou marseillais) et non étrusques.

Cette mainmise laisse quelque peu dans l'ombre les premiers contacts organisés entre le monde méditerranéen et les régions barbares situées à l'ouest et au nord des Alpes : ce sont bien les Étrusques qui ont, les premiers, exploré ces régions avec succès, établissant avec les sociétés indigènes des contacts qui ne sont pas sans conséquence sur l'évolution interne de ces groupes locaux ${ }^{2}$.

C'est dire toute l'importance que revêt l'identification et l'inventaire des rares documents à verser au dossier des importations villanoviennes ou étrusques anciennes au-delà des Alpes. Il s'agit, pour l'essentiel, de vases en bronze retrouvés dans les sépultures les plus riches du Bronze final ou du Hallstatt ancien. Il est hors de doute que la rareté de ces vases dans les contextes de cette époque, le prestige qui devait leur être associé, les réservait à un usage ostentatoire ou religieux ${ }^{3}$. Parmi ces vases, de

1 Elles sont rarissimes: depuis les mises au point récentes (J.-P. Morfi, L'expansion phocéenne en Occident, dix années de recherches (1966-1975), Bulletin de Correspondance Hellénique, 99, 1975, p. 853-896; - In., Le commerce étrusque en France, en Espagne et en Afrique, L'Etruria mineraria, Alti del XII Convegno di Studi Etruschi e Italici, Firenze ... 1979, Florence, 1981, p. 463-508; - M. FEUGÈRE, A. Guillot, Les fouilles de Bragny. 1 : les petits objets dans leur contexte du Hallstatt final, Revue archeologique de l'Est el $d u$ Centre-Est, 37, 1986, p. 207-218), un unique fragment d'anse d'amphore étrusque a èté recueilli sur le site de Lyon-Vaise, Gorge-de-Loup (renseignement C. Bellon), au reste dans le niveau antérieur à la mise en place de la palissade datée par dendrochronologie de 537 avant notre ère. Néanmoins, en dehors de trouvailles qui, en l'ètat actuel des choses, ne changent pas l'image d'une écrasante suprématie grecque sur le commerce rhodanien, on ne peut guère croire locale la trouvaille, mal documentée, d'une coupe de "bucchero nero" presque intacte à Saxon-Sion (Meurthe-et-Moselle) : S. PIÉCHAUd, Le bucchero de Sion (Meurthe et Moselle), in: L'Age du Fer en Lorraine, catalogue d'exposition, Sarreguemines, mai 1987, p. 84 ; - repris par L. Ol.ivifr in : Les Princes celtes et la Méditerranée, Paris, 1988, p. 288, fig. 9.

2 Voir en dernier lieu, B. Chaume, M. Feugère, Les sépultures tumulaires aristocratiques du Hallstatt ancien de Poiseul-la-Ville (Côte-d'Or), $10^{\mathrm{e}}$ suppl. à la Revue archéologique de l'Est et du Centre-Est, Dijon, 1990, p. 53-60.

3 Par exemple G. Chapotat, Le char processionnel de la Côte-Saint-André (Isère), Gallia, 20, 1962, p. 33 sqq.; - ou mieux en mieux connus ${ }^{4}$ figure une série de phiales côtelées en tôle de bronze dont un exemplaire, conservé à Lyon, avait jusqu'ici échappé aux répertoires ${ }^{5}$.

Il s'agit de plusieurs fragments, jointifs, d'un vase écrasé dont la déformation ne permet pas de restituer en toute fiabilité le diamètre ou la hauteur (fig. 1-3). Le bord est conservé sur une longueur de $125 \mathrm{~mm}$; il comporte une lèvre simplement déversée vers l'extérieur et se prolonge par un bandeau vertical de $27 \mathrm{~mm}$, séparé de la panse tronconique par une carène adoucie. Le corps du vase est occupé par de fines cannelures rectilignes, exécutées au repoussé (fig. 3, no 2), dont 23 subsistent partiellement sur les fragments conservés. Le fond du vase manque, une déchirure (bien visible sur le montage de la figure 2) ayant suivi la base inférieure de la panse.

L'examen des divers registres d'inventaire ne pcrmet malheureusement pas de connaître la provenance de cet objet, enregistré dans le nouveau répertoire des bronzes sous le numéro $\mathrm{Br}$. 175-177. L'état de conservation désastreux d'un vase aussi modeste rend très invraisemblable un achat extérieur, à plus forte raison en Italie où l'on pouvait autrefois se procurer à bon compte des vases intacts. L'écrasement et la fragmentation ancienne de cet objet suggèrent au contraire une trouvaille locale, sur un site terrestre.

Les caractéristiques morphologiques permettent de reconnaître les restes d'une phiale étrusque de type Colmar, suivant la dénomination d'O.-H. Frey ${ }^{6}$. Ces coupes en tôle de bronze martelée comptent parmi les premières marchandises exportées par

encore B. Bouloumı́, Le symposion gréco-étrusque et l'aristocratie celtique, in: Les Princes celles et la Méditerranée, Paris, 1988, p. 343-383.

4 Situles de type Kurd : M. EGG, Die hallstattzeitlichen Ilügelgräber bei Helpfau-Uttendorf in Oberösterreich, Jahrbuch des römisch-germanischen Zentralmuseums Mainz, 32, 1985 , p. 323-393; - situles à attaches circulaires : R. DE Marinis, La situla di Trezzo (Milano), in : Varia Archaeologica (Posavski Muzej Brežice, Knjiga 1), Brežice, 1974, p. 67-86.

5 Ce vase figure dans le catalogue de $\mathrm{S}$. Bouchrin et $\mathrm{S}$. Tassinari, Musée de la Civilisation Gallo-Romaine à Lyon. Bronzes antiques, I. Inscriptions, statuaire, vaisselle, Paris, 1976 , sous le $n^{\circ} 168$. La consultation des inventaires anciens et récents des musées lyonnais ne fournit aucune indication de provenance.

6 O.-H. Frfy, Die Entstehung der Silulenkunst. Sludien zur figürlich verzierten Toreutik von Este (R. G. Forschungen, 31), Berlin, 1969, p. 62-63, avec la carte de répartition fig. 35 (liste 3. p. 112); - W. КгммгG, Die griechische Kolonisation im westlichen Mittelmeergebiet und ihre Wirkung auf die Landschaften des westlichen Mitteleuropa, Jahrbuch des römisch-germanischen Zentralmuseums, Mainz, 30, 1983, p. 38. 
PHIALE ÉTRLSQLE À LYON

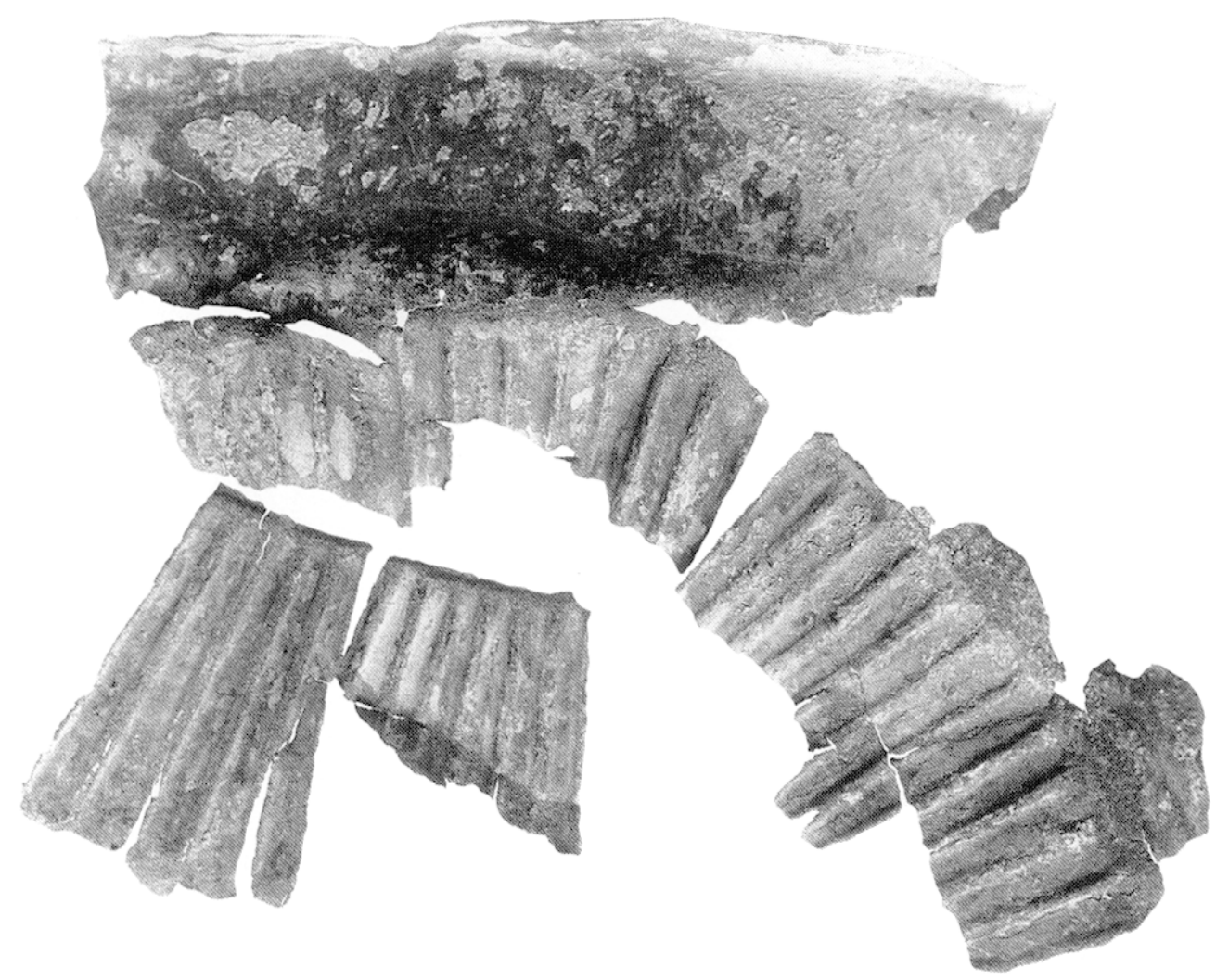

Fig. 1 - Phiale côtelée de Lyon (photo Musée de la civilisation gallo-romaine).

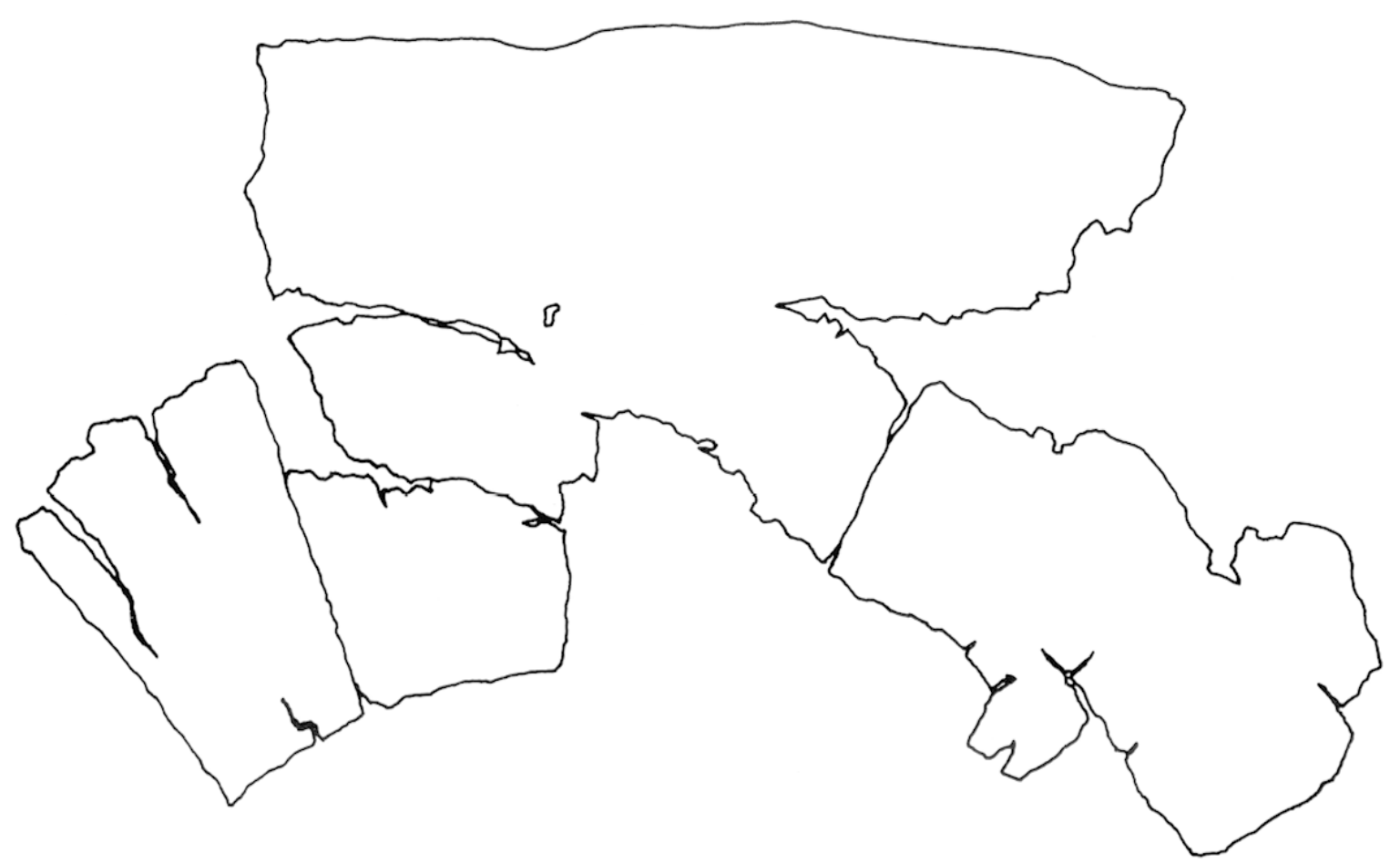

Fig. 2 - Montage des fragments de la figure 1 (noter la déchirure circulaire près de la base). 


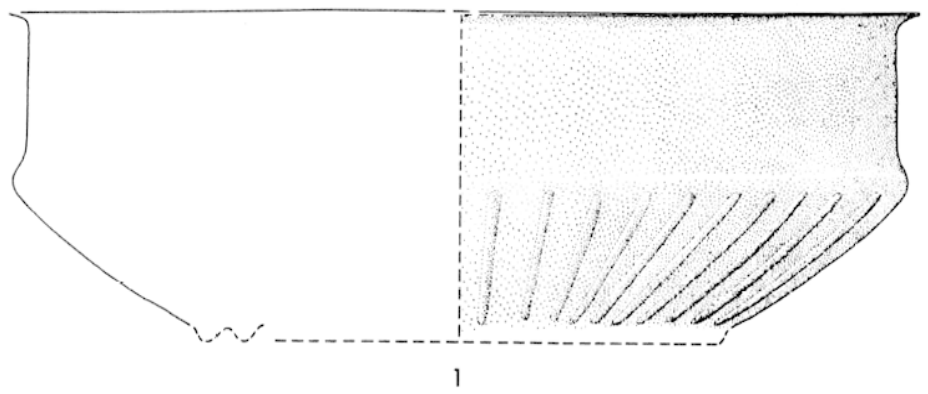

Fig. $3-$

Phiale côtelée de Lyon :

1, profil restitué ;

2 , section transversale au niveau des côtes.
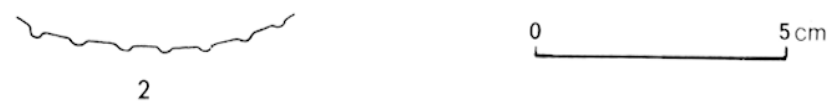

les Étrusques en Italie septentrionale et au-delà des Alpes, dès le début du viI" s. avant notre ère. Leur étude, amorcée par G. Camporeale en 1971, avait montré que cette série particulière, caractérisée par le bandeau cylindrique et les fines cannelures rectilignes (type B de cet auteur), devait être distinguée du groupe, nettement plus nombreux, des phiales à lèvre déversée et grosses côtes arrondies (type A); la comparaison établie entre les découvertes étrusques et les exemplaires retrouvés au Moyen-Orient permet de considérer la plus grande partie des phiales de type A recueillies en Italie comme des importations orientales, alors que la totalité du groupe B semble bien de facture étrusque; l'inventaire dressé par G. Camporeale permettait même d'attribuer à Vetulonia la fabrication des phiales de type $B$ ?

Depuis, affinée par P. H. G. Howes Smith, l'étude a permis de distinguer parmi ces phiales en tôle martelée une vingtaine de groupes dont plusieurs ont été produits en Étrurie : à Vetulonia, mais aussi Cerveteri et Vulci, ainsi que, selon toute vraisemblance, sur d'autres sites d'Italie méridionale ${ }^{8}$.

Très rares au-delà des Alpes (fig. 4), les phiales de type Colmar ne sont jusqu'à présent connues que sur trois sites, où elles apparaissent dans des contextes bien datés du Hallstatt ancien :

7 G. Camporeale, La Tomba del Duce, Florence, 1967, p. 44 sqq.

8 P. H. G. Howes Sмiтh, Bronze Ribbed Bowls from Central Italy and Etruria, Bulletin antieke Beschaving, 59, 2, 1984, p. 73-111; le type B de Camporeale correspond au groupe 10 de Howes Smith, qui confirme sa provenance vétulonienne et propose également d'attribuer à la mème ville ses groupes 8a-c et 9 .
- Francfort, tumulus 12 de Stadtwald (Hesse) (fig. $\left.5, \mathrm{n}^{0} 4\right)^{9}$ : diamètre 158 à $161 \mathrm{~mm}$; hauteur 41 à $45 \mathrm{~mm}, 36$ côtes ;

- Appenwihr, tumulus 1 de Kastenwald (HautRhin) (fig. 5, n $^{\text {os }} 1$ et 2$)^{10}$ : diamètre $132 \mathrm{~mm}$; hauteur $43 \mathrm{~mm}$.

- Poiseul-la-Ville, tumulus 3 de La Perrière (Côte-d'Or) (fig. 5, $\left.\mathrm{n}^{\mathrm{o}} 3\right)^{11}$ : diamètre $163 \mathrm{~mm}$; hauteur $49 \mathrm{~mm}, 61$ côtes.

On observe une relative homogénéité morphologique dans les phiales transalpines de ce groupe, même si certains caractères, comme le nombre des côtes varie presque du simple au double. La phiale de Lyon, dont les dimensions ne peuvent malheureusement pas ètre restituées avec précision, se distingue cependant par sa taille importante : diamètre d'environ $185 \mathrm{~mm}$, hauteur de l'ordre de $62 \mathrm{~mm}$.

Tous les ensembles funéraires ayant livré ce type de phiale témoignent de contacts extrêmement précoces entre l'Étrurie du début de la période orientalisante et le monde indigène. A Francfort, la phiale côtelée est associée à une riche vaisselle de bronze comprenant une situle martelée à attaches circulaires et deux bassins en tôle non décorés. La

9 U. Fischer, Ein Grabhügel der Bronze- und Eisenzeil im Frankfurter Stadtwald (Schriften des Frankfurter Museums für Vor- und Frühgeschichte, IV), Francfort, 1979, p. 69-78; - B. Сhaume, M. Feugterf, op. cit., p. 45, fig. 40.

10 M. Jeht, Ch. Bonnet, Nouvelles fouilles et importantes trouvailles dans la forêt du Kastenwald près de Colmar, Cahiers Alsaciens d'Archéologie, d'Arl el d'Histoire, 1957, p. 24, fig. 7 ; - O.-H. Frey, op. cil., p. 62, fig. 34,1 ;-B. ChaUme, M. Feugère, op. cil., p. 44, fig. 39.

11 B. Chaume, M. Feugère, op. cit., p. 14, fig. 12, no 4 et p. 42 , fig. 38 . 


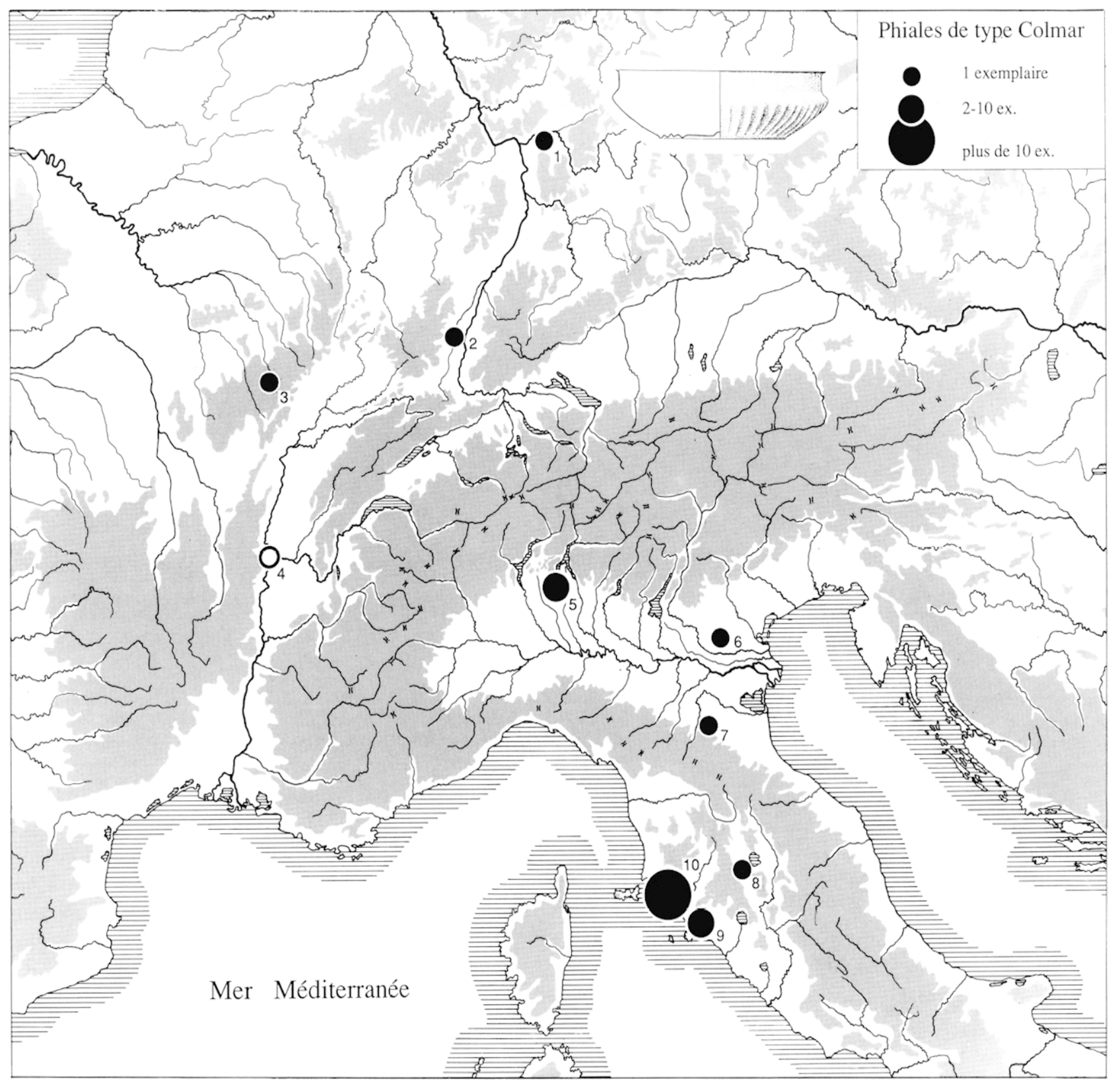

Fig. 4 - Carte de répartition des phiales còtelées de type Colmar (Camporeale B) : 1, Francfort; 2, Appenwihr; 3, Poiseul-la-Ville ; 4, Lyon (?); 5, Còme; 6, Este; 7, Bologne ; 8, Chiusi ; 9, Marsiliana d'Albegna ; 10, Vetulonia.

sépulture a également livré, outre quelques parures (épingles, boutons à bélière), une épée en bronze, un couteau en fer et des objets de harnachement qui témoignent assez du statut aristocratique du défunt, un jeune homme de 25 à 30 ans d'après l'analyse ostéologique ${ }^{12}$.

La célèbre tombe d'Appenwihr, dans la forêt de Kastenwald en Alsace, constitue une sépulture

12 U. Fischer, op. cit.; - voir également B. Chaume, M. FEugÉRE, op. cit., fig. 44. adventice du tumulus I de ce gisement, édifié comme la plupart des autres tertres de cette nécropole (qui semble comprendre au moins une dizaine de monuments funéraires) au Bronze moyen. L'inhumation, fouillée en 1954 et 1955 , a livré un riche mobilier comportant plusieurs bronzes importés. De part et d'autre d'une grande urne en céramique à col déversé et décor peint, d'influence Alb-Salem, se trouvaient deux des offrandes en bronze : une phiale côtelée du type B de Camporeale, et une grande coupe sur pied décorée au repoussé d'une frise animale (de style, si ce n'est de facture, étrusque 


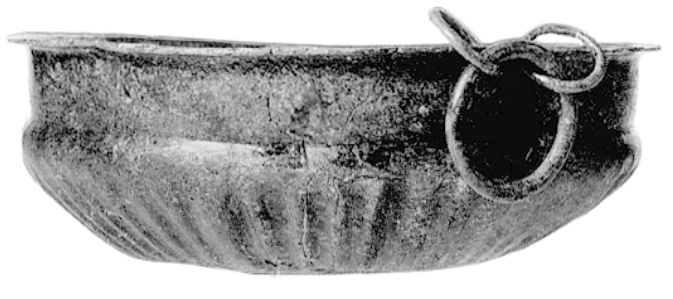

1

0

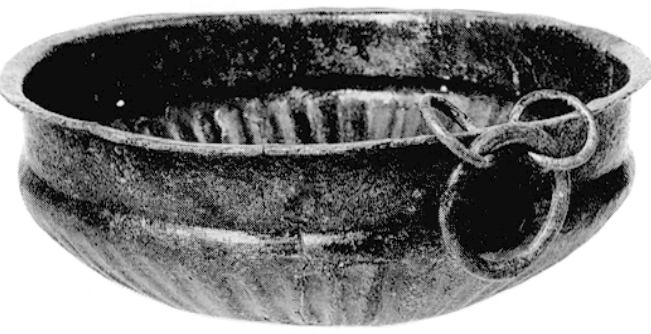

2

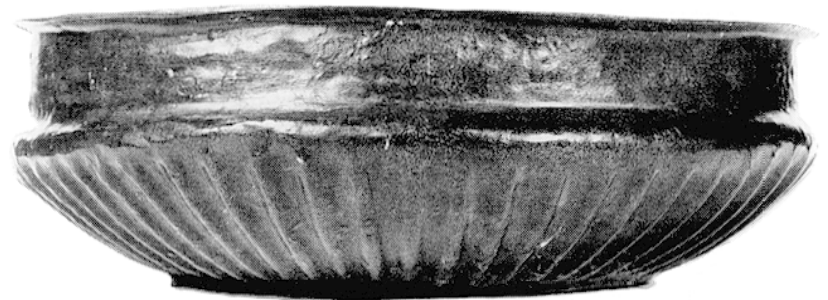

3

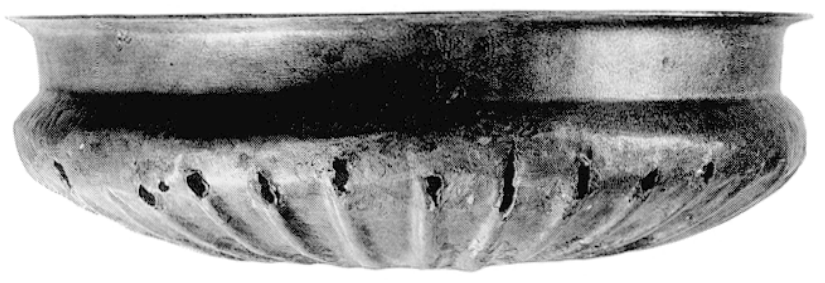

4

Fig. 5 - Phiales étrusques d'Appenwihr (1 et 2), de Poiseul (3) et de Francfort (4) (l'échelle de $3 \mathrm{~cm}$ n'est exacte que pour le $\mathrm{n}^{\circ}$ 1).

ancien $)^{13}$; à l'opposé de la tombe, et donc sans doute près des pieds, on a recueilli une passoire à profil ogival, un petit flacon sphérique formé de deux coques serties, et les restes d'un brasero ou "brûleparfum" à couvercle surmonté d'une fleur de lotus, qui constitue la trouvaille la plus spectaculaire de cette tombe aristocratique. L'ensemble se place probablement dans la première moitié du viI ${ }^{\mathbf{e}}$ s., sans que les travaux anciens ou récents sur les importations de cette sépulture exceptionnelle ${ }^{14}$ aient permis d'en préciser davantage la chronologie.

A Poiseul-la-Ville enfin, une coupe de même type que les précédentes a été recueillie par $R$. Joffroy à l'occasion de ses fouilles de 1977 dans le tumulus 3. L'inhumation, protégée par de grosses pierres, était celle d'un homme enseveli avec sa grande épée en fer. Outre le mobilier classique pour les tombes de cette nécropole correspondant à un groupe social privilégié (épée et rasoirs en fer, bracelet de bronze,

13 Sur la sépulture d'Appenwihr, M. Jehi, Ch. Bonnet, op. cit., p. 19-32; - C. Bonnet, S. Piouin-Mantzer, Sites préhistoriques et protohistoriques fondamentaux de la région colmarienne, Revue d'Alsace, 108, 1982, en particulier p. $20-26$.

14 Pour la pyxide, voir en dernier lieu les observations de Cl. Rol.t.fy, Importations méditerranéennes et repères chronologiques, in : Les Princes celles el la Méditerranée, Paris, 1988, p. $97-98$. perle en terre cuite), le mort était accompagné d'un service à boire importé, déposé à ses pieds: une grande situle en bronze, de type Kurd, à l'intérieur de laquelle on trouva la phiale côtelée. La publication des tumulus de Poiseul-la-Ville ayant donné lieu à une révision chronologique des sépultures à épée du Hallstatt ancien en Bourgogne et en FrancheComté, cette tombe peut être située dans une phase précoce du Premier Age du Fer bourguignon, soit en chronologie absolue aux alentours de 700 avant notre ère ${ }^{15}$.

En Italie, la datation de ces phiales repose sur un grand nombre d'ensembles funéraires, comme par exemple la "Tomba del Carrettino» de Como Ca Morta, que l'on s'accorde maintenant à dater autour de $700^{16}$. Divers exemples de ces coupes peuvent être trouvés dans des contextes de toute la première moitié du vir s. Mais en dehors de l'Italie, nous avons pu montrer que ces importations étrusques s'inscrivent dans une phase ancienne du début de l'Age du Fer, et non tardive ("Ha C2" de G. Kossack)

15 B. Chaume, M. Feugère, op. cit., fig. 38.

16 G. Kossack, Zu den Metallbeigaben des Wagengrabes von Ca'Morta (Como), Sibrium, III, 1956/57, p. 42 et pl. C, d. Dessin de ces phiales dans R. De Marinis, La protostoria, in: Archeologia in Lombardia, Milan, 1982, p. 88, fig. 177. 
comme on pouvait le croire jusqu'alors. On ne peut en effet éviter de rapprocher les bouleversements culturels qui affectent les dernières cultures de l'Age du Bronze, au milieu et dans la seconde moitié du viII ${ }^{e}$ s., de l'émergence de ces premiers contacts et de la multiplication des grandes épées en fer. Ces différents éléments, affectant en moins d'un siècle des sociétés jusque-là plutôt traditionnelles, ne sont certainement pas étrangers les uns aux autres, même si le rôle exact de chaque facteur nous échappe encore en partie.

La phiale de Lyon peut-elle être comptée au nombre des rares documents venant compléter notre information sur cette période encore méconnue? C'est l'hypothèse la plus vraisemblable. Il est évidemment très regrettable qu'aucune note ancienne n'assure la provenance locale de cet objet ${ }^{17}$. Si on accepte le raisonnement ci-dessus, avec ce qu'il comporte de subjectivité, on peut facilement imaginer que ces vestiges, découverts anciennement à Lyon ou dans les environs, ont été considérés jusqu'à aujourd'hui comme une trouvaille gallo-romaine peu significative.

17 La provenance indiquée dans le catalogue imprimé (voir note 5) ne repose que sur une extrapolation, a laquelle
Dans cette hypothèse, la phiale du Musée de Lyon pourrait provenir, elle aussi, d'une sépulture non identifiée du début de l'Age du Fer, passée inaperçue à une époque où la protohistoire ne suscitait pas le même intérêt qu'aujourd'hui ${ }^{18}$.

\section{Michel Feugère}

nous souscrivons en dernier ressort; comme a bien voulu nous le confirmer Fr. Leyge, Conservateur, que nous remercions de son aide, la mention ne repose sur aucun document d'archive conservé ou mentionné au Musée de la civilisation galloromaine.

18 La protohistoire lyonnaise s'est enrichie récemment de nombreuses informations recueillies sur le chantier de Gorge-de-Loup, a Vaisc, mais limitées pour le moment au Hallstatt final et au tout début de La Tène. On consultera notamment, sur ces fouilles : C. Bri.ron, J. Burnouf, J.-M. Martin, Premiers résultats des fouilles sur le site protohistorique de Gorge-de-Loup (Vaise, Lyon, Rhône), Revue archéologique de l'Est et du Centre-Est, 37, 1986, p. 247-251 ; J. Burnouf, C. Bellon, J.-M. Martin el alii, Lyon avant Lugdunum, Un habitat du Premier Age du Fer à Vaise, in : Chr. Goldinfau dir., Aux origines de Lyon, Lyon, 1989, Documents d'Archéologie en Rhòne-Alpes, 2, p. 11-21 (voir également note 1). 